\title{
CHOOSING THE BEST SHOPPING CENTER USING THE MULTI-CRITERIA DECISION METHODS
}

\author{
Kristina Bošković1, \\ Violeta Tomašević ${ }^{*}$ *
}

${ }^{1}$ Kodar Elektromontaža, Belgrade, Serbia

${ }^{2}$ Singidunum University, Belgrade, Serbia

\begin{abstract}
:
This paper discusses the problem of choosing the shopping center which is the most suitable for doing shopping. In modern life circumstances, people constantly have less time available for activities such as shopping, especially in big cities, which gives the great deal of importance to this problem. Considering the fact that there is a large number of shopping centers, as well as many criteria which can directly influence the choice of the place where to go shopping, the problem is becoming more complex, so its solution requires using multi-criteria decision-making methods.

This paper explains two methods used for solving of the considered decision problem: the AHP and the PROMETHEE method. Both of these methods have a strong mathematical background, but also include personal opinion of the decision maker. This implies that the choice of the best shopping center is made considering both objective and subjective factors and is closely linked to the specific decision maker. The differences and similarities in the ranking lists obtained by applying the methods mentioned above, indicate that the results of multi-criteria analysis should be understood as a recommendation, whereas the final decision is still to be made by the deciding person. In order to perform multi-criteria analysis and apply its methods, software developed for that purpose were used.
\end{abstract}

Keywords:

Multi-criteria decision, Alternatives, AHP method, PROMETHEE method, Shopping.

\section{INTRODUCTION}

The complexity of the decision problem primarily depends on the nature of the decision-making system in which decision making is to be performed. Different circumstances and various limitations which exist in the system directly influence the choice of one of the possible alternatives. In the decision-making process, there is often a confrontation between wishes and real needs of the decision maker, on one side, and the available resources, on the other side. What is typical for these resources, is that they are always limited (by type or quantity). Also, when making a decision, several important parameters that characterize each decision should be taken into consideration and those are: the importance of the e-mail:

vitomasevic@singidunum.ac.rs 
decision, the time needed for its making, the costs and the level of complexity.

The decision-making process includes four basic phases: identifying and defining the problem, creating a model, finding solution and implementing solution.

The poblem formulation implies getting an overall picture of the problem (orientation period), after which the components of the problem have to be defined, such as necessary skills the decision maker should have, as well as the decision criteria. Creating a model requires defining a set of possible alternatives, which are to be chosen from. Finding solution, or in other words - choosing the optimal alternative, can be carried out analytically, numerically or by simulation. The last step is the implementation phase, in which the solution obtained in the previous phase is applied to a specific problem.

\section{MULTI-CRITERIA DECISION ANALYSIS}

In many real-world situations [1], [2], in order to achieve the best possible decision, a large number of factors (criteria) needs to be taken into account, so that the decision could be relevant. The criteria can be mutually independent (for example, color and speed) or dependent (age and work experience) and sometimes even mutually opposed, which means that the fulfillment of one criterion can negatively affect the fulfillment of another criterion (price and quantity). Some of the criteria can be expressed quantitatively (weight), while some are described qualitatively (communicativeness). The criteria are usually not equally important to the decision maker, so criteria are often assigned priorities. In such conditions, decision making, which is now seen as multi-criteria decision making, means finding a compromise solution. The purpose of compromise solution is to achieve balance between the criteria, whereas, at the same time, it takes into account the preferences of the decision maker.

The multi-criteria decision-making process begins with the analysis of the problem and the identification of a set of criteria relevant for the decision making. In the beginning of the process, a set of possible alternatives that represent potential solutions to the problem, is also to be identified. Afterwards, an evaluation table is created, which contains values of all criteria for all of the alternatives. Using the evaluation table, the next step is to apply some of the existing multi-criteria decision-making methods (mainly with software support) and obtain the final result: a ranking list of alternatives, from which can be easily seen which alternative is seen as the best.

In this paper, the process described above is illustrated in the example of choosing the best shopping center, using two multi-criteria decision-making methods, the AHP method and the PROMETHEE method, which will be presented in continuation.

\section{THE AHP METHOD}

AHP (Analytical Hierarchy Process, T.L.Saaty [3]) is one of the best-known methods of multi-criteria decision making. It is used in different fields for solving complex and unstructured decision-making problems, when multiple criteria are present [4]. The application of the method starts with structuring the problem, so that hierarchical structure of elements is formed (see Figure 1). The goal, which should be achieved as a result of successful decision making, is placed at the top of the hierarchy. The criteria, deriving from the goal, are to be found at the next level. If a particular decision problem requires, the criteria can be divided into sub-criteria, which are then located at the lower level. In a similar way, sub-criteria can be decomposed into further criteria levels. At the bottom of the hierarchical model, all the available alternatives are placed. Each of these alternatives is seen as the one that potentially meets the defined criteria, and in that way, the overall goal as well.

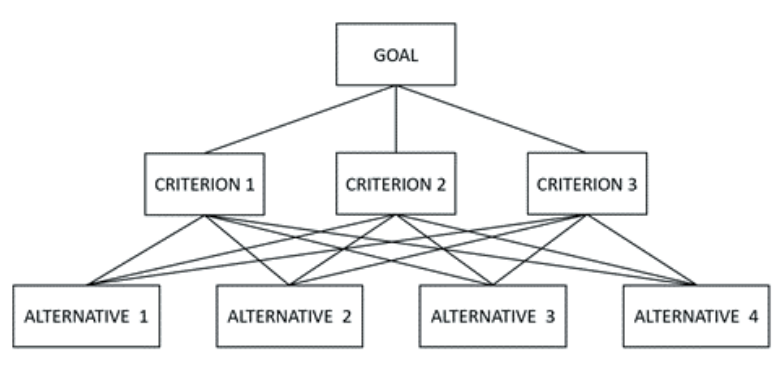

Figure 1 - Hierarchical structure of the problem.

In the second step, the elements in the hierarchy are assigned either numerical or descriptive values, based on which they will be compared. The AHP method consists of parwise comparison of elements at the same hierarchy level, with respect to the common element at the upper level. 
The comparison is performed successively, starting from the first level. Firstly, criteria are compared in pairs, comparing each criterion with another one, whereby these pairs are evaluated with reference to the goal. The procedure is then repeated at each level of the hierarchy and is concluded with the parwise comparison of alternatives, which are to be found at the bottom of the hierarchy. When comparing pairs of elements, a scale of relative importance is to be used, typically Saaty's scale of relative importance. Therefore, when two elements $E_{i}$ i $E_{j}$ are being compared, the importance, that is the priority of one element over another is to be established and, according to the scale applied, the numerical parameter $a_{i j}$ is to be determined. After all pairs have been evaluated, an $n x n$ matrix $A$ is assembled, where $\mathrm{n}$ represents the number of elements at the specific hierarchy level. The elements of the matrix $A$ have the properties given in Equation 1:

$$
\begin{aligned}
& \forall i=1, \ldots, n, \forall j=1, \ldots, n: a_{i j}>0 \\
& \forall i=j: a_{i j}=1 \\
& \forall i=1, \ldots, n, \forall j=1, \ldots, n: a_{i j}=a_{j i}^{-1}
\end{aligned}
$$

Equation 1 - Definition of matrix A.

The next step is to calculate the relative weights of the elements (criteria, sub-criteria or alternatives) in relation to the higher-level element. To achieve that, each parameter $a_{i j}$ of the matrix $\mathcal{A}$ has to be represented as the quotient of the local weights of the elements, that is $a_{i j}=w_{i} / w_{j}$. Using this matrix form, the normalized elements weight vector $w=\left\{w_{1}, w_{2} \ldots w_{n}\right\}^{\mathrm{T}}$ is to be determined by means of one of the following methods: the additive normalization, the eigenvalue/eigenvector method, the logarithmic least squares method, etc.

In the end, the vector $\mathrm{w}$ should be multiplied by the weight coefficient of the higher-level element, with respect to which the comparison was done. This procedure is repeated in sequence, going from higher to lower levels of the hierarchy, until the lowest level with alternatives is reached. The final result, a composite normalized vector for the complete hierarchy, is obtained by multiplying the local weight vectors of each hierarchy level. By means of the composite vector, it is possible to determine the relative priority of alternatives against the goal, which results in their ranking and the choice of the best alternative.

\subsection{THE SUPERDECISIONS SOFTWARE}

For the purpose of quicker and more efficient application of the previously described decision method, multiple software tools which implement AHP method have been developed. In this paper, the SuperDecisions softver [5] was used, created by Saaty, the author of the method.

SuperDecisions software allows the following procedures:

- generating a hierarchical structure of the problem

- pairwise comparison of elements in a hierarchy (by means of matrix or graphically)

- calculation of relative weights of elements

- determination of the ranking of alternatives

- conducting sensitivity analysis which questionates how the change of input data would influence the ranking of alternatives

\section{THE PROMETHEE METHOD}

PROMETHEE (Preference Ranking Organization Method for Enrichment Evaluation, J.Brans [6]) represents a family of six methods that serve for ranking of alternatives, based on multiple criteria. These methods have wide application in different fields (medicine [7], chemistry, banking, etc.), primarily due to their mathematical background, ease of use and the stability of the results achieved by means of them.

The basis of the PROMETHEE method consists in pairwise comparison of available alternatives. The choice of the best alternative doesn't only depend on a set of established criteria and alternatives given, it is moreover influenced by personal beliefs and preferences of the decision maker. Therefore, the solution of a concrete decision problem can't be seen as the best in general, but only as the best according to a specific decision maker.

Let the following multi-criteria problem be given $\max \left\{k_{1}(a), k_{2}(a), \ldots, k_{p}(a) \mid a \in A\right\}$, where A represents a finite set of available alternatives which are to be ranked, whereas $k_{1}, \ldots, k_{p}$ are previously defined criteria. The values of each criterion against each alternative are stored in the evaluation table (see Table 1). 


\begin{tabular}{lcccccc}
\hline Option & $k_{1}$ & $k_{2}$ & $\cdots$ & $k_{j}$ & $\ldots$ & $k_{p}$ \\
\hline$a_{1}$ & $k_{1}\left(a_{1}\right)$ & $k_{2}\left(a_{1}\right)$ & $\ldots$ & $k_{j}\left(a_{1}\right)$ & $\ldots$ & $k_{p}\left(a_{1}\right)$ \\
\hline$a_{2}$ & $k_{1}\left(a_{2}\right)$ & $k_{2}\left(a_{2}\right)$ & $\ldots$ & $k_{j}\left(a_{2}\right)$ & $\ldots$ & $k_{p}\left(a_{2}\right)$ \\
\hline$\ldots$ & $\ldots$ & $\ldots$ & $\ldots$ & $\ldots$ & $\ldots$ & $\ldots$ \\
\hline$a_{i}$ & $k_{1}\left(a_{i}\right)$ & $k_{2}\left(a_{i}\right)$ & $\ldots$ & $k_{j}\left(a_{i}\right)$ & $\ldots$ & $k_{p}\left(a_{i}\right)$ \\
\hline$\cdots$ & $\ldots$ & $\ldots$ & $\ldots$ & $\ldots$ & $\ldots$ & $\ldots$ \\
\hline$a_{q}$ & $k_{1}\left(a_{q}\right)$ & $k_{2}\left(a_{q}\right)$ & $\ldots$ & $k_{j}\left(a_{q}\right)$ & $\ldots$ & $k_{p}\left(a_{q}\right)$ \\
\hline
\end{tabular}

Table 1 - Evaluation table.

On the basis of the evaluation table, the comparison of each pair of alternatives is carried out against each of the criteria. The result of comparing two alternatives $a_{m} \mathrm{i}$ $a_{n}$ against the criterion $k_{j}$ is given as the preference function $P_{j}\left(a_{m}, a_{n}\right)$, whose values are falling to the interval from one to zero $[0,1]$. The value 0 shows the indifference between alternatives, while the value 1 indicates the strong preference of the first alternative over the other one. The six preference functions (Usual, U-Shape, V-Shape, Level, Linear and Gaussian) are defined by the author of the method, which show how the value of the preference function depends on the difference $d_{j}\left(a_{m}, a_{n}\right)$ $=k_{j}\left(a_{m}\right)-k_{j}\left(a_{n}\right)$.

The decision maker, according to his affinities, assignes one of the preference functions to each criterion. Given that the result of comparison of two alternatives $\left(a_{m} \mathrm{i} a_{\mathrm{n}}\right)$ against a criterion $\left(k_{j}\right)$ is the value $\mathrm{P}_{j}\left(a_{m}, a_{n}\right)$, the relation of two alternatives with respect to all criteria can be described by the preference index IP defined by Equation 2, where $w_{j}$ are the relative weights belonging to criteria.

$$
\operatorname{IP}\left(a_{m}, a_{n}\right)=\sum_{j=1}^{p} w_{j} P_{j}\left(a_{m}, a_{n}\right)
$$

Equation 2 - Preference index calculation.

The preference indices are calculated for every pair of alternatives and both values $I P\left(a_{m}, a_{n}\right)$ and $I P\left(a_{n}, a_{m}\right)$ have to be defined. They are then used for either partial (PROMETHEE I) or complete (PROMETHEE II) ranking of alternatives. Therefore, based on the preference indices, three preference flows are calculated as it is given in Equation 3: (1) the positive (outgoing) preference flow $T^{+}(a),(2)$ the negative (incoming) preference flow $T(a)$, and (3) the net preference flow $T(a)$.

$$
\begin{aligned}
& T^{+}(a)=\frac{1}{p-1} \sum_{x * \vec{x}} I P(a, x) \\
& T(a)=\frac{1}{p-1} \sum_{x *} I P(a, x) \\
& T(a)=T^{+}(a)-T^{-}(a)
\end{aligned}
$$

Equation 3 - Preference flows.

The positive or outgoing flow indicates how the alternative $a$ is prefered to all the other alternatives. On the other hand, the negative or incoming flow shows the degree of domination of other alternatives over the alternative $a$. In other words, the positive flow reflects the overall strength, while the negative flow expresses the overall weakness of the alternative. The higher the positive flow, the better the alternative. When it comes to negative flow, the alternative is better with its negative flow being lower. In conslusion, the net preference flow can be seen as the relevant parameter for the complete ranking of alternatives, which means that the alternative choosen as the best will be the one with the highest net flow.

\subsection{THE VISUAL PROMETHEE SOFTWARE}

In this paper, Visual PROMETHEE software [8] has been used for method application. The software was approved by the author of the method and it enables creating a scenario in which the decision maker defines alternatives, criteria and preference functions, based on which alternatives are rated and ranked partially or completely.

The software offers different ways of vizualization of results: PROMETHEE Diamond (two-dimenzional representation in form of angled plane which combines both partial and complete ranking), PROMETHEE Network (a net of nodes in which incomparable alternatives are easy to detect), PROMETHEE Rainbow (shows the final ranking, including contribution of each criterion), GAIA (graphical representation of alternatives and criteria on the GAIA plane, pointing out their mutual relations).

In addition to this, the software also contains a useful tool called Walking Weights, that allows to analyze how the change of criteria weights affects the final ranking of alternatives. In this way, it is possible to determine the intervals in which criteria weights could vary, without causing changes in the ranking of alternatives. 


\section{CASE STUDY}

The multi-criteria decision-making methods described in this paper (AHP and PROMETHEE) have been applied to the problem of choosing the most appropriate shopping center (SC), which is the best for doing shopping or other leisure activities that shopping centers usually offer. The interest for this problem has to do with the fact that life has become fast in modern times, which results in the lack of free time in general, as well as in the lack of time for going shopping. That is why the help with choosing a particular place (shopping center), would be very welcome for an average person.

At the time of writing this paper, the following six shopping centers were considered to be the most visited in Belgrade and therefore choosen as alternatives, based on which the decision making was conducted:

$a_{1}$ Delta City

$a_{2}$ Ušće Shopping Center

$a_{3}$ Stadion Shopping Center

$a_{4} \quad$ Big Fashion Shopping Center

$a_{5}$ Rajićeva Shopping Center

$a_{6}$ Ada Mall

The shopping centers will be compared with respect to the following five criteria:

$k_{1}$ The retail area

$k_{2}$ The car parking options

$k_{21}$ The number of parking spots

$k_{22}$ The parking fee

$k_{3}$ Nearby public transportation options (total number of public tansportation lines)

$k_{4}$ The location (the distance from the city center)

$k_{5}$ The average attendance

$k_{51}$ The attendance on Wednesdays

$k_{52}$ The attendance on Saturdays

As can be seen, the second and the fifth criterion are divided into two sub-criteria. The average attendance includes two sub-criteria as well, which is important because there is a significant difference when it comes to number of visitors on weekdays (in this case, on Wednesday) and on the weekend (on Saturday).

The values for each criterion/sub-criterion for every alternative are given in Table 2. The number of parking spots $\left(k_{21}\right)$ is expressed in hundreds, while the parking fee $\left(k_{22}\right)$ is given in din/h, with the fact that the alternatives $a_{1}$ i $a_{2}$ have no parking fee during the first three hours, whereas the alternative $a_{5}$ includes free parking during the first 30 minutes. The criterion $k_{3}$ refers to the total number of public transportation lines by which the shopping center can be reached. Furthermore, the attendance $\left(k_{5}\right)$ shows the number of shopping center visitors in the period from $5 \mathrm{pm}$ to $8 \mathrm{pm}$, considering location-based check-ins suggested by Google. This criterion is assigned values from 1 to 4 , with 4 expressing the biggest attendance and 1 indicating the smallest number of visitors.

\begin{tabular}{ccccccccc}
\hline & \multirow{2}{*}{$S C$} & $k_{1}$ & \multicolumn{2}{c}{$k_{2}$} & & $k_{4}$ & \multicolumn{2}{c}{$k_{5}$} \\
\cline { 3 - 4 } \cline { 7 - 9 } & $\left(10^{3} \mathrm{~m}^{2}\right)$ & $k_{21}$ & $k_{22}$ & & $(k m)$ & $k_{51}$ & $k_{52}$ \\
\hline$a_{1}$ & 30 & 11 & 100 & 10 & 5.8 & 3 & 4 \\
\hline$a_{2}$ & 50 & 13 & 100 & 22 & 2.9 & 3 & 4 \\
\hline$a_{3}$ & 28 & 0.8 & - & 4 & 7.7 & 3 & 4 \\
\hline$a_{4}$ & 32 & 8 & - & 10 & 4 & 2 & 4 \\
\hline$a_{5}$ & 15.3 & 4.5 & 100 & 9 & 1 & 3 & 4 \\
\hline$a_{6}$ & 34 & 10 & - & 21 & 6.2 & 2 & 4 \\
\hline
\end{tabular}

Table 2 - The values of criteria for all alternatives.

\subsection{APPLICATION OF THE AHP METHOD}

In order to conduct the multi-criteria decision analysis using the AHP method, the hierarchical structure of the problem was created according to the criteria definition given in Table 2 by means of SuperDecisions software.

Afterwards, the alternatives are pairwise compared with respect to a higher-level element, starting from the first level, at which criteria are to be found. If a criterion is diveded into sub-criteria, the comparison of the alternatives (which are at the bottom level) is done against the sub-criteria. If there are no sub-criteria, alternatives are compared with reference to the criteria given. With the aim of comparing the elements, Saaty's nine-point scale was used.

The comparison results were placed in the pairwise comparison matrix. For example, in case of comparing the given alternatives with respect to the number of parking spots, the matrix will have the form shown in Table 3. 


\begin{tabular}{cccccccc}
\hline$k_{21}$ & $a_{1}$ & $a_{2}$ & $a_{3}$ & $a_{4}$ & $a_{5}$ & $a_{6}$ & Priority \\
\hline$a_{1}$ & 1 & $1 / 4$ & 5 & 5 & 7 & 3 & 0.238 \\
\hline$a_{2}$ & 4 & 1 & 6 & 6 & 9 & 5 & 0.470 \\
\hline$a_{3}$ & $1 / 5$ & $1 / 6$ & 1 & 1 & 5 & $1 / 4$ & 0.061 \\
\hline$a_{4}$ & $1 / 5$ & $1 / 6$ & 1 & 1 & 5 & $1 / 4$ & 0.061 \\
\hline$a_{5}$ & $1 / 7$ & $1 / 9$ & $1 / 5$ & $1 / 5$ & 1 & $1 / 7$ & 0.023 \\
\hline$a_{6}$ & $1 / 3$ & $1 / 5$ & 4 & 4 & 7 & 1 & 0.147 \\
\hline
\end{tabular}

Table 3 - The comparison of alternatives with respect to the number of parking spots.

Based on all pairwise comparison matrices, the SuperDecisions software generated the ranking of the alternatives as shown in Figure 2.

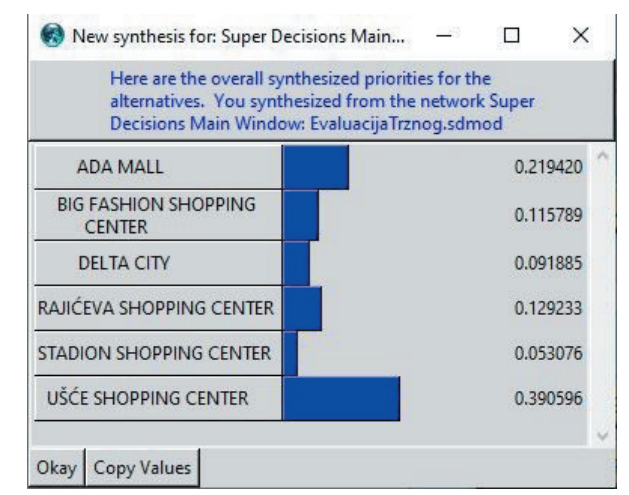

Figure 2 - The ranking of alternatives using the AHP method.

\subsection{APPLICATION OF THE PROMETHEE METHOD}

The application of the PROMETHEE method to the considered decision problem using Visual PROMETHEE software starts with defining the scenario and its parameters that is the input data related to alternatives and criteria given in Table 2.

Compared with the AHP method, the criteria are now organized in a different way. Namely, the criteria are classified considering some of their common features. The groups of criteria can then be grouped into clusters. In this particular example, the sub-criteria defined in the hierarchy can be seen as criteria, which are then grouped, depending on their nature.

Firstly, it was defined whether a particular criterion has to be minimazed or maximazed. If a criterion is quantitative, it was expressed in a suitable unit of measurement.
In order to rate criteria, appropriate evaluation scales were defined for qualitative criteria (yes/no, 5-point scale and 4-point scale, depending on specific criterion).

The weights of the criteria are the same as the ones calculated by means of the AHP method. For each criterion, a preference function was defined using the appropriate tool available in Visual PROMETHEE software (the decision maker fills out a questionnare based on which their affinities are determined).

The alternatives were grouped into two categories depending on whether the shopping center was opened in the last five years or earlier.

Using the data entered by decision maker, the software calculates the partial ranking of alternatives (PROMETHEE I method), which shows that SC Ušće has the highest positive and the smallest negative flow, so it is certainly the best alternative. It can be noticed that there are two pairs of alternatives which are incomparable.

Figure 3 shows the complete ranking of alternatives (PROMETHEE II method), in which the obtained ranking is based on the net flow.

$$
\begin{aligned}
& \forall i=1, \ldots, n, \forall j=1, \ldots, n: a_{i j}>0 \\
& \forall i=j: a_{i j}=1 \\
& \forall i=1, \ldots, n, \forall j=1, \ldots, n: a_{i j}=a_{j i}^{-1} \\
& I P\left(a_{m}, a_{n}\right)=\sum_{j=1}^{p} w_{j} P_{j}\left(a_{m}, a_{n}\right) \\
& T^{+}(a)=\frac{1}{p-1} \sum_{x *} I P(a, x) \\
& T(a)=\frac{1}{p-1} \sum_{x *} I P(a, x) \\
& T(a)=T^{+}(a)-T(a)
\end{aligned}
$$

\begin{tabular}{|c|c|c|c|c|c|}
\hline \multicolumn{4}{|c|}{ PROMETHEE Flow Table } & - & $x$ \\
\hline Rank & \multicolumn{2}{|c|}{ akcija } & \multirow{2}{*}{$\begin{array}{r}\text { Phi } \\
0,6008\end{array}$} & \multirow{2}{*}{$\begin{array}{r}\text { Phit } \\
0,6275\end{array}$} & \multirow{2}{*}{$\begin{array}{r}\text { Phi- } \\
0,0267\end{array}$} \\
\hline 1 & Ušce & $\square$ & & & \\
\hline 2 & Ada Mall & [ & 0,2382 & 0,3509 & 0,1127 \\
\hline 3 & Big Fashion & [ & $-0,0638$ & 0,1290 & 0,1928 \\
\hline 4 & DC & $\square$ & $-0,1706$ & 0,0785 & 0,2491 \\
\hline 5 & Stadion & $\square$ & $-0,2969$ & 0,0634 & 0,3604 \\
\hline 6 & Rajiceva & 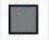 & $-0,3076$ & 0,1225 & 0,4301 \\
\hline
\end{tabular}

Figure 3 - The complete ranking using the PROMETHEE II method. 


\section{CONCLUSION}

From the ranking results obtained by the application of the AHP and the PROMETHEE decision-making methods, it can be seen that the alternative SC Ušće is the best in both cases, followed by Ada Mall, whereas there are slight differences in the ranking of other alternatives. The main reason for that is the fact that personal beliefs, foresights and experiences of the decision maker are differently modelated in each of these two methods. Therefore, it would be possible to obtain different rankings even if the same method would be applied by two decision makers (for example, in case of PROMETHEE method, based on the affinities of the decision makers, two different scenarios could be defined, including different preference functions). What can be concluded is that each decision could be seen as good enough, provided that it uses the algorithm of multiple criteria decision making. Certain requirements and opinions of the specific decision maker are also a crucial part of making a potentially good decision

In order to make the best possible decision, these two methods can be interconnected, so that each of them can use other method's results [9]. For example, in this paper the weights of the entities were determined by applying the AHP method, which were then used for ranking of alternatives by means of PROMETHEE method. The use of available programs simplifies the entire decisionmaking process, since the results are generated straight away.

One of the biggest advantages of both methods is the possibility to perform a sensitivity analysis, which shows how changes in importance of criteria influence the ranking of alternatives.

\section{REFERENCES}

[1] M. M. de Brito and M. Evers, "Multi-criteria decision-making for flood risk management: a survey of the current state of the art," Natural Hazards and Earth System Sciences, vol. 16, pp. 1019-1033, 2016.

[2] I. Zafar, I. Y. Wuni, G. Q. Shen, H. Zahoor and J. Xue, "A decision support framework for sustainable highway alignment embracing variant preferences of stakeholders: case of China Pakistan economic corridor," Journal of Environmental Planning and Management, 2019.

[3] T. L. Saaty, "Decision making with the Analytic Hierarchy Process," International Journal of Services Sciences, vol. 1, pp. 83-98, 2008.
[4] A. U. Khan and Y. Ali, "Analytical hierarchy process (AHP) and analytic network process methods and their applications: a twenty year review from 20002019," International Journal of the Analytic Hierarchy Process, 2020.

[5] Creative Decisions Foundation, "SuperDecisions," 2021. [Online]. Available: http://www.superdecisions.com/tutorials/.

[6] J. P. Brans and B. Mareschal, "PROMETHEE methods," in Multiple Criteria Decision Analysis: State of the Art Surweys, New York, Springer, 2005, pp. 163-195.

[7] F. S. Yildirim, M. Sayan, T. Sanlidag, B. Uzun, D. U. Ozsahin and I. Ozsahin, "Comparative Evaluation of the Treatment of COVID-19 with Multicriteria Decision-Making Techniques," Journal of Healthcare Engineering, 2021.

[8] "Visual PROMETHEE 1.4 Manual," VPSolutions, 2013. [Online]. Available: http://www.prometheegaia.net/files/VPManual.pdf. [Accessed 2021].

[9] J. Draginčić i V. Marković, „Kombinovanje metoda AHP i PROMETHEE pri izboru najpogodnijeg rešenja oblaganja kanala za navodnjavanje," Vodoprivreda, t. 45, pp. 275-280, 2013. 